\title{
Ablauf und Ergebnisse des Besuches einer chinesischen Delegation in Wildau
}

\author{
Prof. Dr. Bernd Wonneberger, Dipl.-Psych. Hans Könecke, Dr. Bernd Lehne
}

\begin{abstract}
Auf Einladung des Präsidenten der Technischen Fachhochschule Wildau, Herrn Prof. Dr--Ing. W. Arlt, besuchte in der Zeit vom 9. bis 13.11.1997 eine hochrangige Delegation von Managern der chinesischen Kohleindustrie die Technische Fachhochschule in Wildau. Der Delegation unter Leitung des Generalsekretärs der China Coal Enterprices Management Association, Wang Guangde, gehörten sieben Direktoren und Ökonomen von Unternehmen der Kohleförderung und -verarbeitung aus verschiedenen Provinzen Chinas an. Dieser Besuch war das Ergebnis der vielfältigen Aktivitäten des Instituts für Internationales Marketing und Unternehmensfuihrung unter Leitung von Prof. Dr. Wonneberger zur Markterschließung fuir brandenburgische mittelständische Unternehmen in Südostasien, insbesondere in China, in enger Verbindung von studentischer Ausbildung und in Kooperation mit Unternehmen. Die Kontakte entstanden im März 1996 während eines Besuches von Prof. Wonneberger in Beijing und Xian.
\end{abstract}

Ziel des Besuches der chinesischen Delegation (welche auf eigene Kosten anreiste) war das Kennenlernen der Leistungsfähigkeit der technischen und betriebswirtschaftlichen Forschungsinstitute der Technischen Fachhochschule Wildau und der Technischen Universitäten in Cottbus und Berlin, der Erfahrungsaustausch über die Umstrukturierung ehemals großer staatlicher Unternehmen auf die Erfordernisse von Marktwirtschaft und Wettbewerb und moderne Methoden effizienter Unternehmensführung. Auf dieser Grundlage bereitete das Institut für Internationales Marketing und Unternehmensfuihrung in enger Zusammenarbeit mit der Delegationsleitung seit dem Frühjahr 1997 ein Besuchsprogramm vor, das in Zusammenarbeit mit der Lausitzer Braunkohle AG und den Technischen Universitäten Cottbus und Berlin realisiert wurde.

Am 9.11.1997 landeten unsere acht chinesischen Gäste nach fünfzehnstündigem Flug von Beijing über Frankfurt/ M. um 17.35 Uhr in Berlin-Tegel. Das Besuchsprogramm begann am Montag, dem 10.11.1997, mit der Begrüßung durch den Präsidenten der Technischen Fachhochschule Wildau, Prof. Dr.-Ing. W. Arlt, der das Bundesland Brandenburg, die Technische Fachhochschule Wildau sowie ihr Technologietransfer- und Weiterbildungszentrum vorstellte. Der Leiter der chinesischen Delegation, Generalsekretär Wang, bedankte sich sehr für die Einladung und das Besuchsprogramm, von dem er zahlreiche Anregungen für die Entwicklung und Modernisierung der chinesischen Kohleindustrie erwartete. Nach den Begrüßungsworten führte der Präsident unsere Gäste auf einem mehrstündigen Rundgang durch die Hochschule, zu- nächst am Standort Bahnhofstraße durch das Hochschulrechenzentrum, das Institut für Unternehmenslogistik und durch das China-Institut. Nach einem kurzen Bustransfer wurden am Standort Friedrich-Engels-Straße das Labor für Oberflächentechnik, das Institut für Wasserund Abwassertechnik, das Institut für Innovation und Verfahrenstechnik und das Institut für Qualitätsmanagement besichtigt.

Am Nachmittag des 10.11.1997 stießen dann die Vorträge zweier Referenten auf lebhaftes Interesse unserer Gäste. Dr. Badke, Geschäftsführer der Sondermaschinenbau Wildau $\mathrm{GmbH}$, berichtete ausführlich über seine $\mathrm{Er}$ fahrungen bei der Umstrukturierung der TAKRAF AG und der SMB Wildau GmbH. Es wurde deutlich, wie innerhalb weniger Jahre aus riesigen Kombinaten überschaubare Unternehmen wurden, welche ihre Aktivitäten (und den Mitarbeiterstamm) auf ihre Kernkompetenzen konzentrieren. In Ergänzung zum Referat von Dr. Badke sprach Prof. Dr. Arlt die „Freisetzung“ tausender von Mitarbeitern und die damit verbundenen sozialen Probleme an.

Durch die Konzentration auf ihre Kernkompetenzen werden unter Umständen selbst Unternehmen mit wenigen Dutzend Mitarbeitern wettbewerbsfähig auf dem Weltmarkt. Danach machte Prof. Dr. Biermann in seinem Vortrag deutlich, wieweit das moderne Management eines Unternehmens hinsichtlich der Schlüsselmotive Qualität, Kosten, Zeit und Innovation im Wettbewerb steht. Die außerordentlich zahlreichen Fragen an die beiden Referenten fokussierten dann vor allem die sozialen und politischen Dimensionen jenes Prozesses der wirtschaftlichen Umstrukturierung, der in den vergangenen sieben Jahren in den neuen Bundesländern vollzogen wurde. Viele Erwerbstätige wurden und werden für neue Tätigkeiten weitergebildet oder umgeschult. Andere Erwerbstätige gingen in den vorgezogenen Altersruhestand, oder sie werden in Maßnahmen des sogenannten zweiten Arbeitsmarktes beschäftigt. Sehr viele fanden bis heute keinen neuen Arbeitsplatz auf dem ersten Arbeitsmarkt, was sich in der Statistik der Bundesanstalt für Arbeit nur zum Teil widerspiegelt. Die Maßnahmen der Weiterbildung, Umschulung oder Arbeitsförderung werden Jahr für Jahr mit Milliardensummen aus den Kassen des Bundes sowie der Europäischen Union bezuschußt - Unterstützungen, die der Volksrepublik China schlicht fehlen. Eine konsequente Rationalisierung der chinesischen Staatsbetriebe würde 100 bis 200 Mio. Arbeitssuchende "freisetzen“ (die Spannweite der Prognosen hierzu ist groß), ohne daß dieser Prozeß durch ein dem deutschen vergleichbares System der Arbeitslosen- oder Rentenversicherung ab- 
gefedert werden könnte. Alle Teilnehmer dieser Diskussion - ob Chinesen oder Deutsche - waren sich daher einig in dem Resumee, daß ein eigener „chinesischer Weg" entwickelt und realisiert werden sollte, welcher Erfahrungen des modernen Managements mit den sozialen Erfordernissen der Volksrepublik China verknüpft.

Am Dienstag, dem 11.11.1997, fand ein dreieinhalbstündiges Treffen zwischen unseren chinesischen Gästen und 22 brandenburgischen Unternehmen statt. Als weitere Repräsentanten der brandenburgischen Wirtschaft nahmen an diesem Treffen auch der stellvertretende Hauptgeschäftsfuihrer der IHK Cottbus, Dr. Kotzorek, der Geschäftsführer von Seed Capital Brandenburg, Dr. Groß, und der Geschäftsfuihrer der T.IN.A. Brandenburg GmbH, Prof. Dr. Schulze, teil. Als Vertreter des Wirtschaftsministeriums überbrachte Dr. Schilling die Grüße des brandenburgischen Wirtschaftsministers. Unter der Moderation von Prof. Dr. Wonneberger stellten einige der teilnehmenden Unternehmen innovative Lösungen der Meßtechnik, der Nachrichtentechnik oder des Umweltschutzes vor. Andere Unternehmen schilderten ihre Erfahrungen aus der jahrelangen Arbeit mit chinesischen Partnern u. a. bei Projekten des Kraftwerkbaus oder der Verkehrstechnik. Insgesamt wurde nach Auffassung der Autoren deutlich, daß die brandenburgische Wirtschaft etliche interessante technische Beiträge und Lösungen für die Weiterentwicklung der chinesischen Kohleförderung, Kohleverarbeitung und Energieerzeugung zu liefern vermag. Generalsekretär Wang bedankte sich entsprechend bei den brandenburgischen Unternehmen für ihr zahlreiches Erscheinen und die Präsentation ihres Leistungsvermögens.

Trotz des nebligen Novemberwetters konnten unsere Gäste am Mittwoch, dem 12.11.1997, einen ganztägigen Ausflug zur LAUBAG und zur Brandenburgischen Technischen Universität (BTU) Cottbus genießen. Im Tagebau Jänschwalde besichtigten sie eine Abraumförderbrücke, welche urspïnglich von der TAKRAF errichtet

wudau (MAZ), Eine
hochrangige Delegation
von Managern der chine-
sischen Kohleindustrie
weilt seit gestern an der
Technischen Fachhoch-
schule Wildau. Morgen
findet ein Treffen mit
Vertretern von zwanzig
Brandenburger und Berli-
ner Unternehmen aus
verschiedenen Wirt-
schaftsbereichen statt. Es
sollen Mbglichkeiten ei-
ner Kooperation mit der
chinesischen Kohleindu-
strie ausgelotet werden.
Auf dem Programm ste-
hen auch Besuche der
Lausitzer Braunkohle
AG und der Technischen
Universitäten Berlin be-
ziehungsweise Cottbus.

Märkische Allgemeine Zeitung für Brandenburg vom 10.11.1997 worden war und nun in den vergangenen Jahren u. a. von $A B B$ Cottbus modernisiert wurde. Vertreter von LAUBAG und ABB erläuterten diese Modernisierung. Danach wurde eine Dichtwand besichtigt, die das Eindringen von Grundwasser in die Grube des Tagebaues verhindert. Einige der chinesischen Bergwerke/Tagebauunternehmen hatten sich bereits vor der Reise stark für diese Technologie interessiert. Am Nachmittag wurde dann die Fakultät
IV der BTU Cottbus besucht. Prof. Dr. Hüttl, zugleich Vizepräsident der BTU Cottbus, referierte für den Bereich Umwelt- und Verfahrenstechnik insbesondere über Aspekte des Bodenschutzes und der Rekultivierung von Tagebauen. Die Fachgespräche dieses Tages wurden, nach einer Besichtigung und Führung durch die BTU Cottbus, bei einem gemeinsamen Abendessen in einer Spreewaldgaststätte zu Ende geführt.

Am Donnerstag, dem 13.11.1997, verließen unsere Gäste Wildau nach dem Austausch von zahlreichen Geschenken. Trotz aller Tücken des Berliner Stadtverkehrs erschien die Delegation fast pünktlich bei Prof. Dr. Wolff im Institut für Angewandte Geowissenschaften der Technischen Universität Berlin. Dieser stellte seine Arbeiten vor, insbesondere die vielfältigen und langjährigen Kontakte zur VR China, und gab einen Überblick über Aufbau und Struktur der TU Berlin. Nach einem letzten gemeinsamen Mittagessen verabschiedeten die Autoren ihre Gäste um 14 Uhr am Flughafen BerlinTegel, von dem aus die Delegation weiterflog zu Geschäftsverhandlungen in Belgien und Frankreich.

\section{Ergebnisse des Besuches und Perspektiven der Beziehungen zur chinesischen Kohle- industrie}

Generalsekretär Wang sagte am Schluß des Treffens mit den brandenburgischen Unternehmern, daß er die vorgestellten Leistungen der Unternehmen den über 100 Mitgliedsunternehmen seines Verbandes weiterempfehlen werde. Der stellvertretende Hauptgeschäftsführer der IHK Cottbus, Dr. Kotzorek, formulierte im Namen der brandenburgischen Wirtschaft den Wunsch, die Beziehungen rasch und zum gegenseitigen Vorteil auszubauen. Als ein wichtiges Instrument hierfür nannte er den Austausch von Praktikanten auf allen Ebenen Studentenaustausch ebenso wie der Austausch von Auszubildenden, Mitarbeitern und Führungskräften aus allen Ebenen eines Unternehmens. Er betonte weiter, bei der Entwicklung der Geschäftsbeziehungen zwischen brandenburgischen und chinesischen Unternehmen der Kohleindustrie unbedingt frühzeitig auch die Finanzierung der Geschäfte zu klären.

Die Autoren dieses Beitrages hoffen, daß sich die Finanzierungen von Geschäften mit der chinesischen Kohleindustrie relativ leicht lösen lassen. Dies gründen wir darauf, daß die Fragen der Energieerzeugung und -verteilung in der Wirtschaftspolitik der VR China höchste Priorität haben. In der Produktion sowie dem Export von Steinkohle und auch von Braunkohle ist die VR China Weltmarktführer mit einer Jahresfördermenge von 1.300 Mio t Steinkohle. Um diese Stellung halten und ausbauen zu können, ist der Einsatz modernster Technologien notwendig, so daß spezielle Lösungen brandenburgischer Anbieter stets von Interesse sind. Hierfür werden ggf. auch von chinesischer Seite vergleichsweise rasch Devisen und Devisenkredite bereitgestellt, wie die Autoren aus eigener Erfahrung in Beijing wissen. 
Bei der offiziellen Verabschiedung am Donnerstag früh sprach Generalsekretär Wang eine Einladung an Prof. Dr. Arlt und Prof. Dr. Wonneberger zum Besuch in Beijing Ende Februar 1998 aus. Wir hoffen, diese Einladung rasch annehmen und die Reise nach China organisieren zu können, um dort, gemeinsam mit interessierten brandenburgischen Unternehmen, die Entwicklung der Geschäftsbeziehungen und den Praktikantenaustausch voranbringen zu können.

\author{
Verfasser \\ Prof. Dr. Bernd Wonneberger \\ Dipl.-Psych. Hans Könecke \\ Dr. Bernd Lehne \\ Technische Fachhochschule Wildau \\ Institut für Internationales Marketing und \\ Unternehmensfuihrung \\ Fachbereich Wirtschaft, Verwaltung, Recht \\ Bahnhofstraße \\ 15745 Wildau \\ Tel. + 49 (0) 3375 - 508325 \\ Fax $+49(0) 3375-508388$
}

\section{Gespräche in fachchinesisch an der Fachhochschule}

Chinesische Delegation weilt in Wildau und interessiert sich für Kohleabbau und Privatisierung von staatlichen Unternehmen

Wildau (MAZ). Hoher Besuch steht der Technischen Fachhochschule von Wildau seit Sonntag ins Haus. Eine zehnköpfige Delegation von führenden Managern der chinesischen Kohleindustrie hălt sich noch bis morgen in Wildau auf, um in Gesprächen die Kontakte zu intensivieren und die künftige $\mathrm{Zu}$ sammenarbeit auszubauen.

China ist das Land mit der weltweit größten Kohleförderung. Im Zentrum der Gesprắche steht deshalb das Thema der Kohlegewinnung. Daneben wollen die Gastgeber aber auch ihre Kenntnisse zur Privatisierung und Umstrukturierung ehemals staatseigener Betriebe darlegen. „Dieses Thema wird in China zunehmend interessanter, und wir wollen unsere guten beziehungsweise schlechten Erfahrungen, die wir auf diesem Gebiet ge- macht haben, weitergeben", sagt Hans Könecke, der als wissenschaftlicher Mitarbeiter beim Institut für Internationales Marketing und Unternehmungsführung das Programm mitgestaltet hat. Das Institut ist Teil der Technischen Fachhochschule, das sich insbesondere mit dem Technologietransfer beschäftigt.

Mit dem Besuch will man auch die wirtschaftliche Kooperation zwischen den beiden Staaten ankurbeln. Aus diesem Grund hatten die Gastgeber gestern Vertreter von 23 Brandenburger und Berliner Unternehmen - sie bieten Techniken zur Kohleförderung und -veredelung an - in die Răume der Fachhochschule zu einem $\mathrm{Ge}$ spräch mit den chinesischen Gästen geladen.

Dies ist der erste Besuch einer chinesischen Delegati- on an der Fachhochschule von Wildau. Erste Kontakte bestehen bereits seit einigen Jahren. Dazu zăhlt ein derzeit laufendes Forschungsprojekt des Instituts für Internationales Marketing und Unternehmungsführung, das Institutsmitglieder nach $\mathrm{Chi}$ na geführt hat. Ziel dieses Projekts ist es, mittelständischen Firmen aus Branden. burg den suddostasiatischen Markt zu erschließen.

Auch auf studentischer Ebene bestehen seit einigen Jahren Kontakte. 1993 absolvierten Wildauer Studenten erstmals ein halbjähriges Praktikum bei einer deutschen Firma in China. Zuvor hatten ein zweijăhriger Sprachunterricht und ein sechswöchiger Intensivkurs vor Ort auf dem Programm gestanden. Mit dem Besuch sollen die Kontakte intensiviert werden. gabs

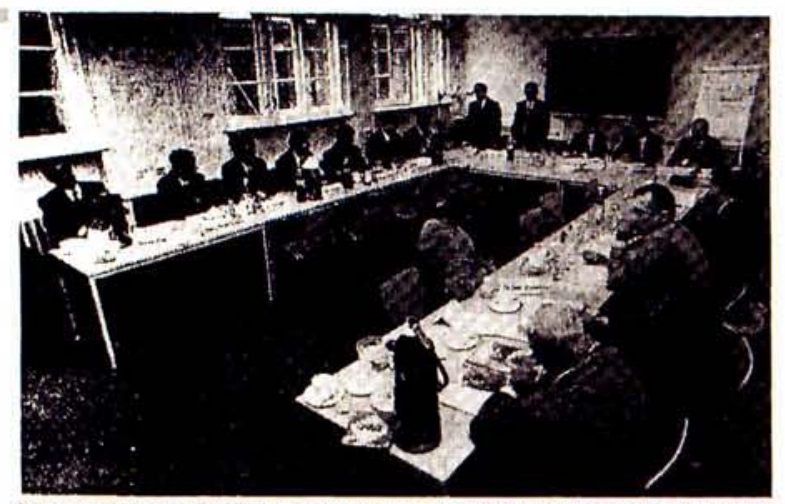

